\title{
A randomized controlled trial of venlafaxine ER and paroxetine in the treatment of outpatients with panic disorder
}

Mark Pollack • Richard Mangano $\cdot$ Richard Entsuah • Evan Tzanis • Naomi M. Simon • Ying Zhang

Published online: 23 November 2007

(C) Springer-Verlag 2007

Erratum to: Psychopharmacology

DOI: 10.1007/s00213-007-0821-0

Ying Zhang should be included in the following article as an author.

Ying Zhang

Senior Biostatistician,

Neuroscience,

Wyeth Research,

Collegeville, Pennsylvania, USA

The online version of the original article can be found at http://dx.doi. org/10.1007/s00213-007-0821-0.

M. Pollack $(\square) \cdot$ N. M. Simon

Massachusetts General Hospital, Simches Research Building,

185 Cambridge Street, Suite 2200, 2nd Floor,

Boston, MA 02114-2790, USA

e-mail: mpollack@partners.org

R. Mangano $\cdot$ R. Entsuah $\cdot$ E. Tzanis $\cdot$ Y. Zhang

Neuroscience,

Wyeth Research,

Collegeville, PA, USA 